\title{
LOS JUEGOS ANCESTRALES COMO ESTRATEGIA, DESDE RELATOS BIOGRÁFICOS, PARA LA VALORACION DESÍ Y EL RECONOCIMIENTO DEL OTRO EN AGROPARQUE LOS SOCHES
}

\section{ANCESTRAL GAMES AS A STRATEGY, FROM BIOGRAPHICAL STORIES, FOR THE VALUATION OF SELF AND THE RECOGNITION OF THE OTHER IN AGROPARK LOS SOCHES}

\section{Alexander Gómez Uribe ${ }^{1}$ \\ Astrid Ramírez Valencia ${ }^{2}$ \\ Universidad Distrital Francisco José de Caldas Bogotá}

\begin{abstract}
$1 \quad$ Estudiante en formación de la licenciatura en Pedagogía Infantil con énfasis en la primera infancia, Universidad Distrital Francisco José de Caldas, Docente en formación en diferentes instituciones educativas de primaria y secundaria, privadas. aramirezv@udistrital.edu.co ORCID https://orcid.org/0000-0001-9640-2183
\end{abstract}

2 Docente-investigadora, Universidad Distrital Francisco José de Caldas Bogotá, Colombia, Ph.D. en Lenguaje y cultura, formadora de profesores de inglés durante más de 25 años. Investigadora y maestra en la Universidad Distrital Francisco José de Caldas. correo aramirezv@udistrital.edu. co ORCID: https://orcid.org/0000-0002-3025-5982

\section{RESUMEN}

El juego es, por excelencia, una vivencia infantil. Niños y niñas comparten durante éste su propia identidad, en tanto ponen en acción manifestaciones de la cultura que han subjetivizado, como, por ejemplo: creencias, formas de convivencia, manera de interrelacionarse, ética para con los otros, entre otras. Los niños y niñas del Agroparque Los Soches juegan y, en esa medida, se aprovechan 
los juegos ancestrales o tradicionales que llevan a cabo para ejecutar una estrategia pedagógica en la cual jueguen y expongan a través de relatos biográficos las experiencias vividas durante el juego, a los fines de impulsar la reflexión de sí como valoración y el otro como reconocimiento del otro, caras de la misma moneda de la identidad cultural.

\section{PALABRAS CLAVE:}

juego ancestral, relato biográfico, estrategia pedagógica, valoración de sí, reconocimiento del otro.

\section{ABSTRACT}

The game is, by excellence, a child's experience. During play, boys and girls share their own identity, as they put into action manifestations of the culture, they have subjectivized, such as: beliefs, ways of coexistence, ways of interrelating, ethics towards others, among others. The children of Agroparque Los Soches play and, to that extent, the ancestral or traditional games they play are used to execute a pedagogical strategy in which they play and expose through biographical stories the experiences lived during the game, in order to promote the reflection of themselves as valuation and the other as recognition of the other, sides of the same coin of cultural identity.

\section{KEY WORDS:}

ancestral game, biographical story, pedagogical strategy, self-appraisal, recognition of the other.

\section{INTRODUCCION}

La investigación tiene por objetivo proponer la estratega didáctica denominada Los juegos ancestrales como estrategia, desde relatos biográficos, para la valoración de sí y el reconocimiento del otro en el Agroparque los Soches, la cual surge desde la mediación pedagógica con los niños y niñas del Agroparque los Soches, ubicado en la localidad de Usme Rural, en el marco de la Práctica Formativa de la línea de investigación Subjetividades, Diversidad y Estética de la licenciatura en Pedagogía Infantil de la Universidad Distrital Francisco José de Caldas.

Esta propuesta está encaminada a la reflexión sobre la propia identidad y la de los otros a través del juego ancestral, desde los relatos biográficos, que permiten indagar la historia de cada niño y niña, de modo que se reconozca su valor cultural e identitario $y$, en consecuencia, el de otro con quien juega, en tanto se asume que el juego es una posibilidad cultural donde las interacciones permiten la circulación de la cultura y, por tanto, la propia diversidad cultural.

En consecuencia, el punto de partida educativo son las relaciones interpersonales de los niños y niñas en las cuales ocurren el diálogo y el intercambio de tradiciones cargadas de lógicas y perspectivas diferentes; como el juego es normativo y lúdico a la vez se generan relaciones basadas en el respeto mutuo de los individuos desde la diversidad cultural y social, lo cual conduce a la convivencia; en este caso entre los niños y niñas del Agroparque los Soches.

Esta propuesta pedagógica teleológicamente sirve para abrir un espacio a la reflexión propositiva del juego como estrategia, alrededor de los conceptos: juego ancestral o tradicional, relato biográfico, valoración de sí y reconocimiento del otro y pedagogía intercultural. Esta concepción se hace en función de propiciar experiencias pedagógicas en donde el juego sea el centro de las mismas, ya que al jugar se comparten significados y se transforman elementos de la cotidianidad que conforman las identidades de los niños y les permiten valorar su identidad y reconoce las de los otros. 
La etapa de la niñez es propicia para la reflexión de lo que se ha subjetivizado como sujeto dentro de un contexto y la dimensión constitutiva de éste donde se constituye al sujeto mismo (Fornet-Betancourt, 2009). Ello porque esta reflexión permite comprender los procesos que se están viviendo en la medida en que compartimos espacios donde las creencias, las formas de convivencia, la manera de interrelacionarnos, la ética para con los otros, entre otras manifestaciones culturales. Siendo el juego una de los espacios por excelencia de la niñez resulta propicio usarlo con intención pedagógica, en el marco de una pedagogía cultural, porque la pregunta por ¿qué y cómo jugamos? es, en esencia, la pregunta por lo que somos como sujetos culturales.

\section{PERSPECTIVA TEÓRICA}

\section{JUEGOS ANCESTRALES O TRADICIONALES}

El juego tradicional será parte de ese conjunto donde se guarda el reservorio que asienta la familia y, entonces, nuestros parientes, madres, padres, abuelas o abuelos, tías o tíos, y nanas desde su oralidad nos llevan todo el universo popular a través de diversos géneros de tradición oral como fruto de aquella herencia del pasado.

Entonces, el juego ancestral o tradicional aflora -como la gastronomía, la música popular, los ritos, las supersticiones, las leyendas, entre otros- como expresión cultural que pone de manifiesto el patrimonio cultural al cual se pertenece y el cual se nos hereda. Según la Organización de las Naciones Unidas para los Refugiados (ACNUR) (2017) al ser heredados de generación en generación representan la manera como se dedica tiempo en una cultura al ocio y el entretenimiento; pero también, como mientras se juega se aprende acerca de solidaridad, respeto por la diferencia y convivencia.
Al respecto, Saco (2001) afirmó que el juego ancestral o tradicional que se transmite de generación en generación, usualmente en forma de tradición oral, da cuenta del proceso histórico de un pueblo, esto es su circular simbólico cultural. Cuando se reconozco cómo éste me hace a mí un sujeto de una determinada cultura -razón por la cual juego lo que juego y cómo esto ha sido jugado por mis generaciones precedentes-, cuando me comprendo a mí mismo mientras reflexiono acerca de cómo me relaciono con los demás y desde ello convivo, logro alcanzar identidad. Larrain (2003) al respecto estableció que la identidad es un discurso o narrativa sobre sí mismo construido en la interacción con otros mediante ese patrón de significados culturales. Así que mientras se juega, se interacciona, se expresa cómo juego yo tal o cual, escucho cómo lo hacen los otros, consensuamos la manera de jugar y las reglas de unos y otros se está construyendo identidad.

El juego es entonces una posibilidad de expresión de la identidad, en la cual la propia identidad se reconoce y valora desde la diferencia. En esta última opera el proceso de subjetivación desde la cultura y con ello se reconoce que un aspecto fundamental de la identidad es, según Marañón Rodríguez (2011), el de "basarse en un conjunto de prácticas, costumbres, cosmovisiones y representaciones heredadas $y$ que son valoradas como expresiones significantes de lo que un grupo es en sí mismo" (p. 03). Además de ser expresión cultural presente, también es expresión cultural del pasado, ya que lo que se juega ha sido transmitido de esa manera por generaciones que también lo han hecho así, por lo tanto, el juego retrotrae un tiempo anterior en el presente, en una imagen de historia condensada.

He aquí que durante el juego la identidad se moviliza en la interacción con los otros para la construcción de una auto-imagen y de una narrativa personal (Larrain, 2003). 
Mientras los niños y niñas juegan se envuelven permanentemente en la red de lo heredado y en ello se construye y mantiene su cultura, pero además se la recrea y la renueva con su propia experiencia vital y la de otros, procesos interculturales que enriquecen la cultura de cada cual.

\section{RELATO BIOGRÁFICO}

Es un texto donde ocurre el reconocimiento de la historia personal como fuente de información que muestra la experiencia singular de la persona en su dimensión temporal (Sancho, 2014). Por ello, según Sancho (2014), el relato biográfico es un tipo de texto donde se narra la vida de una persona desde su propia voz, por lo que hace posible su historia de vida como una forma de subjetivación en la que el sujeto puede narrarse, contarse, pensarse.

En el caso de la identidad, donde el sujeto reconoce quién es, qué ha heredado, qué prácticas culturales lo conducen a actuar $\mathrm{y}$, por ello, de quién se diferencia, qué no es su herencia y cuáles prácticas culturales no son las suyas, el relato biográfico es un medio de autorreconocimiento identitario y también de diferenciación.

En la investigación, los relatos biográficos serán entonces un espacio de expresión de sí para narrar lo vivenciado durante la interacción social del juego, durante el cual se valora la propia identidad y la del otro para la diferenciación cultural, lo cual convoca indefectiblemente a la valoración de la identidad, en ese juego incesante de identidad y diferencia.

\section{RECONOCIMIENTO DEL SÍ Y DEL OTRO}

La Organización de las Naciones Unidas para la Educación, la Ciencia y la Cultura (UNESCO) (2008) concibe este concepto como el contacto cultural donde ocurre la toma de conciencia del valor positivo de las diferencias como enriquecimiento y oportunidad de reflexión en espacios intermedios de negociación y encuentro.

En consecuencia, dentro del ámbito educativo, la UNESCO (2008) propone el desarrollo de acciones en las cuales se favorezca el contacto cultural con inclusión, con la participación de todos en cohesión social, con vitalidad y en paz. Así que ¿qué mejor espacio de contacto cultural que el juego ancestral o tradicional?

En una estrategia pedagógica, donde el juego ancestral sea el centro, se reconoce que los sujetos culturales tienen diferentes procedencias culturales y, a la vez, que éstas se ponen a actuar en interculturalidad, con lo cual la acción educativa asume una tarea doble -impulsada por UNESCO (2008)-: la toma de conciencia de la diferencia cultural que trae como consecuencia la valoración de mí mismo y el reconocimiento del contacto cultural como enriquecimiento y oportunidad de negociación y encuentro, no necesariamente exento de conflictos

\section{PEDAGOGÍA INTERCULTURAL}

Una pedagogía de la alfabetización biográfica se hace necesaria en la educación de niños y jóvenes. Según Fornet-Betancourt (2009) esta pedagogía es necesaria, toda vez que a pesar de estar inmersos en contextos de plena interculturalidad la manera que hemos subjetivizado para interrelacionarnos con los otros, el sujeto que se ha hecho de nosotros es uno con prejuicios, sin diálogo cultural y con contactos culturales de segregación y diferencia, sujeto que el autor nominó como inclinados.

En esa medida, una pedagogía intercultural conduce biografía y de lo que he heredado y aprendido como sujeto de manera que pueda hacer una ruptura con ese contacto cultural como diferencia; esta pedagogía es entonces orientadora de la calidad del ser 
humano que incorpora la pluralidad de saberes a su comprensión del mundo de manera que se construya su conciencia humana.

En esta investigación, en consecuencia, jugar lo que ancestralmente se ha heredado es una actividad lúdica ciertamente, pero también es una actividad donde aprendo acerca de mí mismo, de mi proceso de subjetivización cultural para reconocer qué se ha hecho de mí para relacionarme con los otros y con ello cumplir lo que Fornet-Betancourt (2009) promueve como pedagogía intercultural: un crecimiento "para ser cada día más 'humanos', más equitativos y para aprender a vivir juntos" (p. 6).

\section{MÉTODO}

El enfoque de investigación cualitativa (IC) es pertinente con el objetivo de investigación, porque se da valía a cada actor social (Sánchez, 2000), en tanto es un ser social único que recibe la cultura de su pueblo y su familia y que la resignifica y la recrea de acuerdo a su propia experiencia. Por lo que se puede comprender el fenómeno (de la valoración de sí y del reconocimiento del otro) desde el marco de referencia que dan los propios actores sociales dentro del contexto, tal y como lo establecen Cook y Reichardt (2003).

Ello implica que la investigación lleva hacia la inmersión en la realidad, en trabajo de campo, por lo que debe establecerse "una interacción entre los objetivos y la realidad de la situación de campo; observar y recolectar los datos directamente de la realidad, en su situación natural" (Balestrini, 2002, p. 132)

En este marco de producción de conocimiento, el juego ancestral o tradicional desde relatos biográficos es justamente el foco investigativo de la Metodología Historia de vida (Sancho, 2014). Su búsqueda es la narración que permite expresar los componentes de la subjetividad para comprender los fenómenos sociales que ocurren mientras se juega.

En el campo de la educación la investigación biográfico-narrativa introduce cambios a partir de la generación de diarios y relatos con lo que reivindica, metodológicamente, el ejercicio de dejar oír la voz de los sujetos como forma de representación de la realidad. Estos se convierten "en individuos biográficos con capacidad de acción y elaboradores activos del conocimiento y de las visiones sobre el mundo" (p. 26), en particular cuando la identidad, siendo auto-imagen y narrativa personal es la búsqueda pedagógica para el aprendizaje, medularmente, actitudinal de sí y del otro.

\section{RESULTADOS}

La propuesta pedagógica diseñada tiene la siguiente caracterización:

Nombre de la actividad: Juegos ancestrales, desde relatos biográficos para la valoración de sí y el reconocimiento del otro en Agroparque los Soches

Situacion problemática a intervenir: son dos las problemáticas que generaron esta propuesta pedagógica, a saber:

1. El desencuentro que se produce entre los niños y niñas de la zona urbana y de la zona rural dentro del espacio escolar, lo que en ocasiones genera un choque cultural y divisiones en las aulas y genera la desvalorización de sí y el desconocimiento del otro.

2. El uso de los medios tecnológicos y su influencia en los gustos de los niños y niñas que desplaza la existencia del juego ancestral o tradicional dentro de la cultura de los niños y niñas con la consecuente desenfatización de la identidad. 


\section{POBLACIÓN}

En la intervención asistieron 20 niños y niñas que se encontraban en el rango de los 4 a 15 años de edad; los niños y niñas provienen de diferentes sectores: sector de las piedras, sector de mangueras, sector de rincón grande, sector del Uval. Todos los niños y niñas estudian en el Colegio Rural EI Uval.

Rol del estudiante: Participa activamente en los juegos tradicionales para generar, posteriormente, relatos biográficos en los cuales valore la diferencia entre su propia identidad, como reconocimiento de sí y del otro.

Rol del profesor: Genera la reflexión de la diversidad cultural a través del juego ancestral o tradicional para la socialización de la diferencia y la producción de relatos biográficos para la reflexión cultural.

Actúa desde el marco de la pedagogía intercultural para promover la alfabetización biográfica como vía para la alfabetización biográfica que convoca a convivir juntos y desarrollarnos como humanos.

Juegos privilegiados: los propios niños y niñas seleccionaron los juegos, a saber: juego de muñecas, juega la rana, juego de carros, juego tejo, correr en el campo de la vereda del Agroparque

\section{PROCEDIMIENTO}

La actividad didáctica fue actividad lúdica en esencia, toda vez que el juego era su foco. Los contenidos conceptuales se refirieron a la identidad y al reconocimiento de sí y del otro (UNESCO, 2008); los contenidos desarrollados fueron:

$\checkmark$ Conceptuales: específicamente referidos a juego ancestral o tradicional, identidad cultural, diferencia cultural, interculturalidad. $\checkmark$ Procedimentales: que específicamente desarrollaron la interacción social con su consecuente relación afectiva como campo experiencial, además del aprendizaje de lo normativo social como organización, reglas y roles;

$\checkmark$ Actitudinales: contenidos actitudinales para aprender acerca del respeto, del encuentro y la valoración cultural.

La reflexión cultural, tras los juegos, fue la acción mediadora docente, a los fines del autorreconocimiento de la propia identidad y de la diferencia con el otro alcanzada desde el desarrollo de los contenidos. Ello condujo a la producción de los relatos biográficos que expresaban las reflexiones de los niños y niñas del AgroParque los Soches acerca de sí mismos y de los otros niños y niñas con la mirada puesta en la identidad, la diferencia y la diversidad cultural.

\section{DISCUSIÓN}

Hallazgos primarios: dos hallazgos emergieron como una constante: la preferencia de los niños y niñas de jugar en el campo que representa el Agroparque, toda vez que el lugar les simboliza la naturaleza, el aire libre, el espacio abierto, la presencia de animales. Jugar corriendo, como lo han hecho todos los niños y niñas que ancestralmente han vivido allí es una experiencia con la naturaleza. En segundo lugar, la valoración de la socialización, el encuentro con los otros niños y niñas mientras se juega, el contacto entre todos; este último hallazgo es producto de comparar la vida en pandemia y aislamiento (que viven los niños de la ciudad) y la vida en libertad aun y cuando haya pandemia de los niños del Agroparque que es una zona rural.

En consecuencia, según los relatos de los niños y niñas no se trata esencialmente del juego y de lo que se hereda, aun y cuando 
sí logró constatarse que pueden reconocer el impacto de los medios tecnológicos en sus vidas, especialmente de los niños de la ciudad. Así como la comparación entre esa vida infantil y la vida del juego en el campo como una experiencia de libertad y naturaleza que unánimemente valoran.

El juego en el campo (correr es lo que prefieren) y vivir la experiencia de observarlo y sentirlo mientras lo hacen y jugar como experiencia de contacto y encuentro cultural los hace reconocer la diferencia entre la vida del niño en la ciudad y el campo y que cada cual reconozca la vida del otro $y$, sin embargo, todos terminan por preferir el juego en el campo.

Otros juegos ancestrales o tradicionales que emergieron como idea fueron los sempiternos juegos de muñecas y carros que manifiestan la diferenciación de género. En este caso el juego ancestral se posesiona como una posibilidad cultural de construcción de rol de género, donde niños y niñas inician los procesos sociales de diferenciación y asunción del género.

El juego es experiencial, es una vivencia en la que mientras hay esparcimiento, divertimento, ocio y entretenimiento se aprende, de allí su suma valoración dentro de los procesos didácticos en loa que se tiene determinado un tipo de aprendizaje (Ramos, Gutiérrez y Mancilla, 2015). En esa medida, los aprendizajes se convierten en significativos, porque quedan grabados como una experiencia placentera. Los juegos llevados a cabo durante la ejecución en el Agroparque Los Soches fueron ciertamente experiencias vivenciales de la vida en el campo, de la comparación de esa vida y juego en la ciudad; también fortalecieron una vuelta al juego que no tecnológico, sino ancestral: muñecas y carros aparecieron para que los niños y niñas fueran historia condensada de lo por siempre hicieron niños y niñas en el pasado.
En consecuencia, pedagógicamente, el juego es realmente una posibilidad cultural en el marco de una pedagogía intercultural que permite a los niños y niñas retrotraer su pasado, valorar la diferencia, privilegiar el juego en el campo y encontrarse sin distingos, ni diferencias.

\section{CONCLUSIONES}

Si bien es cierto que la identidad, en el incesante juego de diferencia y reconocimiento, se forja en la vida misma, dentro del hogar, en las prácticas cotidianas que acaecen en y con la familia, en las interacciones sociales dentro de los contextos a los cuales se pertenece, también es cierto que la educación formal tiene la obligación de ofrecer espacios académicos donde la identidad se forme.

Ello por la naturaleza social latinoamericana donde niños y niñas se forman como sujetos inclinados que se desencuentran y alejan culturalmente, tal es la experiencia de los niños y niñas del Agroparque Los Soches, quienes se forman como niños citadinos o rurales y por lo cual tiene un contacto cultural de división. Las experiencias pedagógicas como la ejecutada pueden, en consecuencia, provocar una ruptura de ese desencuentro, generar un nuevo vínculo y lazo social que produzca nuevas subjetivización de quiénes son mientras juegan y de la necesidad de acercarse alorando quiénes son y reconociendo quienes son los otros.

Los relatos biográficos, en un marco de socialización, aseguraron la conciencia de lo que debían valorar y reconocer: a sí mismos y a los otros como otros con otras culturas a las cuales respetar. La interacción fue armónica, por la conciencia de lo que se hacía; también la contrastación de vida en la ciudad y en el campo en un diálogo cultural con contactos culturales sin segregación ni diferencias, sino convivencia y negociación. 


\section{REFERENCIAS BIBLIOGRÁFICAS}

Balestrini, M. (2002). Cómo se elabora el proyecto de investigación para los estudios formulativos o exploratorios, descriptivos, diagnósticos, evaluativos, formulación de hipótesis causales, experimentales $y$ los proyectos factibles. ( $6^{a} \mathrm{Ed}$.). Caracas: Consultores Asociados.

Cook, T., Reichardt, Ch. (2005) (5 $5^{\mathrm{a}}$ Ed.) Métodos cualitativos y cuantitativos en investigación evaluativa. Madrid: Morata

Fornet-Betancourt, R. (2009).Interculturalidad en procesos de intersubjetivización. Reflexiones. México: Coordinación General de Educación Intercultural y Bilingüe

Larrain, J. (2003) El concepto de identidad. Revista FAMECOS, No 21, pp. 3042. http://www.revistas.univerciencia. org/index.php/famecos/article/ viewFile/348/279

Marañón Rodríguez, J.L. (2011) Reflexiones teóricas acerca de la interrelación entre memoria histórica e imaginarios sociales Revista Contribuciones a las Ciencias Sociales www.eumed.net/rev/ cccss $/ 121$

Mieles Barrera, M. D., Tonon, G., Alvarado, S. (2012). Investigación cualitativa: el análisis temático para el tratamiento de la información desde el enfoque de la fenomenología social. En impreso: Universitas Humanística, núm. 74, juliodiciembre, 2012, pp. 195-225. Pontificia Universidad Javeriana, Colombia. Revista Electrónica Redalyc. http:// www.redalyc.org/pdf/791/79125420009. pdf
Organización de las Naciones Unidas para la Educación, la Ciencia y la Cultura (2008). Educación y Diversidad Cultural: Lecciones desde la práctica innovadora de América Latina. Santiago de Chile: Autor

Organización de las Naciones Unidas para los Refugiados (ACNUR) (2017). Juegos tradicionales del mundo: forma de expresión cultural. https://eacnur.org/ blog/juegos-tradicionales-del-mundoformas-expresion-cultural/

Ramos, R., Gutiérrez, E., Mancilla, M. (2015). El juego como estrategia didáctica en la práctica docente en estudiantes de nivel básico. XIII Congreso Nacional de Investigación Educativa. http:// www.comie.org.mx/congresol memoriaelectronica/v13/doc/0709.pdf

Sancho, J. (2014). Historias de vida: el relato biográfico entre el autoconocimiento y dar cuenta de la vida social. Revista Praxis Educativa, Vol. 18, №2, pp. 2433, http://www.fchst.unlpam.edu.ar/ojs/ index.php/praxis/

Sánchez, E. (2000). Todos con la esperanza. Caracas: UCV. FHE 